\title{
Increasing Top-Down Mass Spectrometry Sequence Coverage by an Order of Magnitude through Optimized Internal Fragment Generation and Assignment
}

\author{
Nicholas D. Schmitt,1,3 Joshua M. Berger, 1,3 Jeremy B. Conway, 1,3 Jeffrey N. Agar1,2,3,* \\ ${ }^{1}$ Department of Chemistry and Chemical Biology, Northeastern University, Boston, MA 02115, United States \\ ${ }^{2}$ Department of Pharmaceutical Sciences, Northeastern University, Boston, MA 02115, United States \\ ${ }^{3}$ Barnett Institute for Chemical and Biological Analysis, Northeastern University, Boston, MA 02115, United States
}

\section{Contents:}

Figure S1. Evaluation of FSD $b$-ion generation of SOD1 ${ }^{\mathrm{G} 93 \mathrm{~A}}$ as modulated by declustering potential

Figure S2. Internal fragment ion assignment scheme

Figure S3. Ions assigned and Mascot identification scores evaluated by declustering potential

Figure S4. Ambiguity comparison of three model proteins by mass accuracy alone

Figure S5. Internal fragment ion frameshift ambiguity

Table S1. Calculated ambiguity data of four model proteins

Table S2. Fragmentation coverage and ions generated when using terminal fragments alone vs. using terminals and internals 

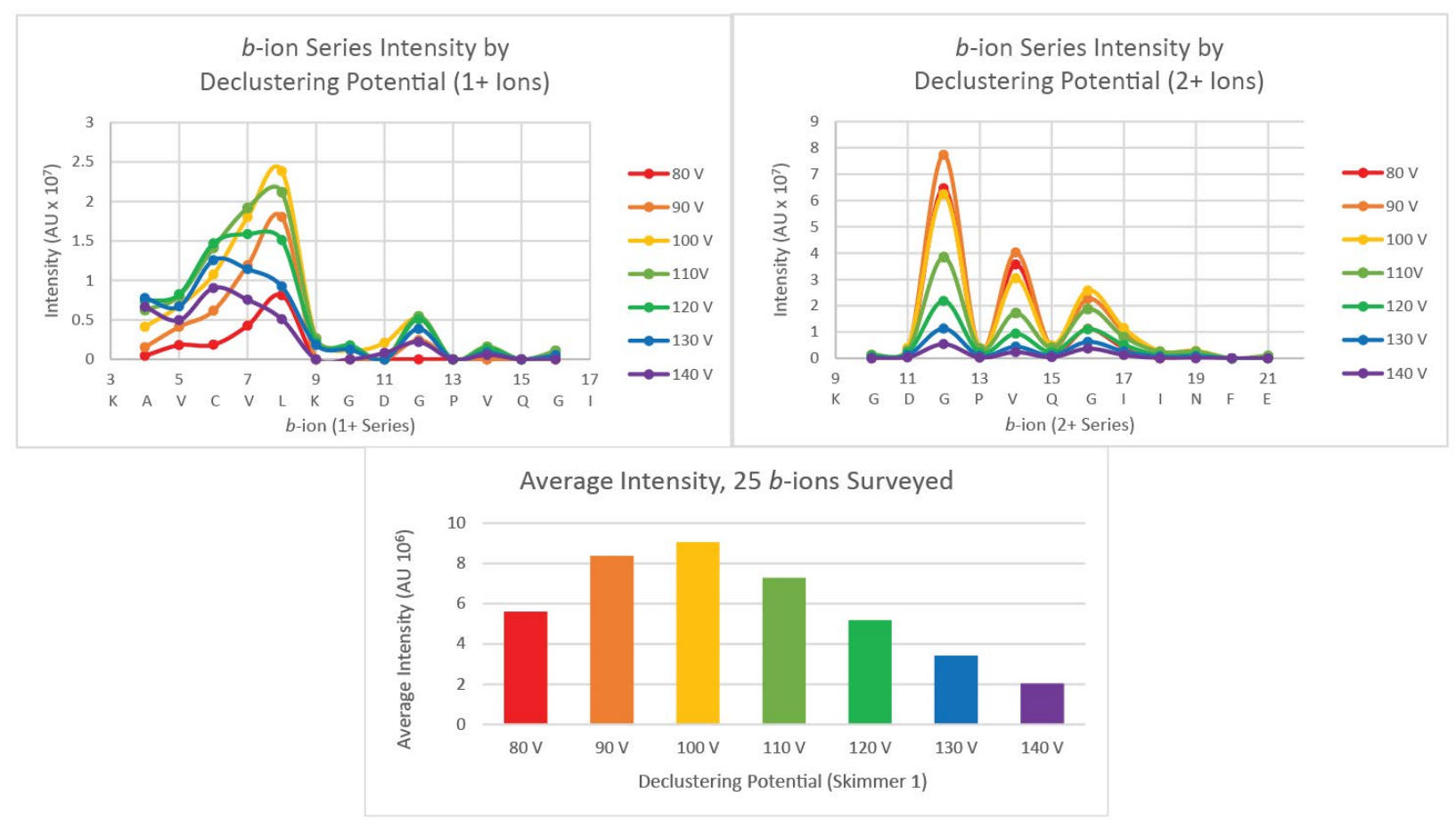

Figure S1. Evaluation of FSD $b$-ion generation of SOD1G93A as modulated by declustering potential (Skimmer 1). Top Left and Top Right: $1+$ and 2+, respectively, $b$-ion series intensities as they change with skimmer 1 voltage. Bottom: Bar graph of the sum of these intensities in both plots. These are the ions used for internal calibration. 


\section{Internal Fragment lon Assignment Scheme}



Figure S2. Internal fragment ion assignment scheme with percentages representing 1ppm mass error tolerance (for other ambiguity ratios, see Figure 1 and Figure S4). Depicted is a workflow for achieving ion assignment for all internal fragment ions, which represented $87 \%$ of identified ions in our collisional dissociation experiments. Depending on the mass accuracy of the measurement and therefor the mass error tolerance used in fragment ion assignment search, a population of fragment ions will be unambiguous and be able to be assigned immediately ( $\sim 80 \%$ at $1 \mathrm{ppm}$ mass error tolerance for a protein up to $30 \mathrm{kDa}$ ). Remaining ambiguous ions can be parsed by the rules we describe into three categories: frameshift ambiguous, arrangement ambiguous, or mass accuracy ambiguous. The portion of mass accuracy ambiguous fragments will depend on the mass accuracy of the measurement as depicted in Figure 1 and Figure S4. Frameshift and arrangement ambiguous fragments will be ambiguous always, even if perfect mass accuracy were achieved. Frameshift ambiguous fragments can be locally assigned (overlapping sequence assignment), or fragmentation propensity rules can be applied to all classes of ambiguous fragments to determine a putative assignment. $\mathrm{MS}^{3}$ can be used to definitively assign all internal fragments. Candidates for internal fragment ions can be generated using top-down proteomics software such as Mascot TD (Matrix Sciences). In Mascot, an instrument setting must be chosen or created which includes internal fragments (type yb and ya). The resulting list of hits can be exported and sorted to determine which fragment matches are ambiguous, and then refined according to the rules we set forth in the associated manuscript to assign correct matches and eliminate incorrect matches. If the protein being studied has a known sequence, creating a custom database containing only that protein can result in much faster searches and ease access to internal fragment data. 

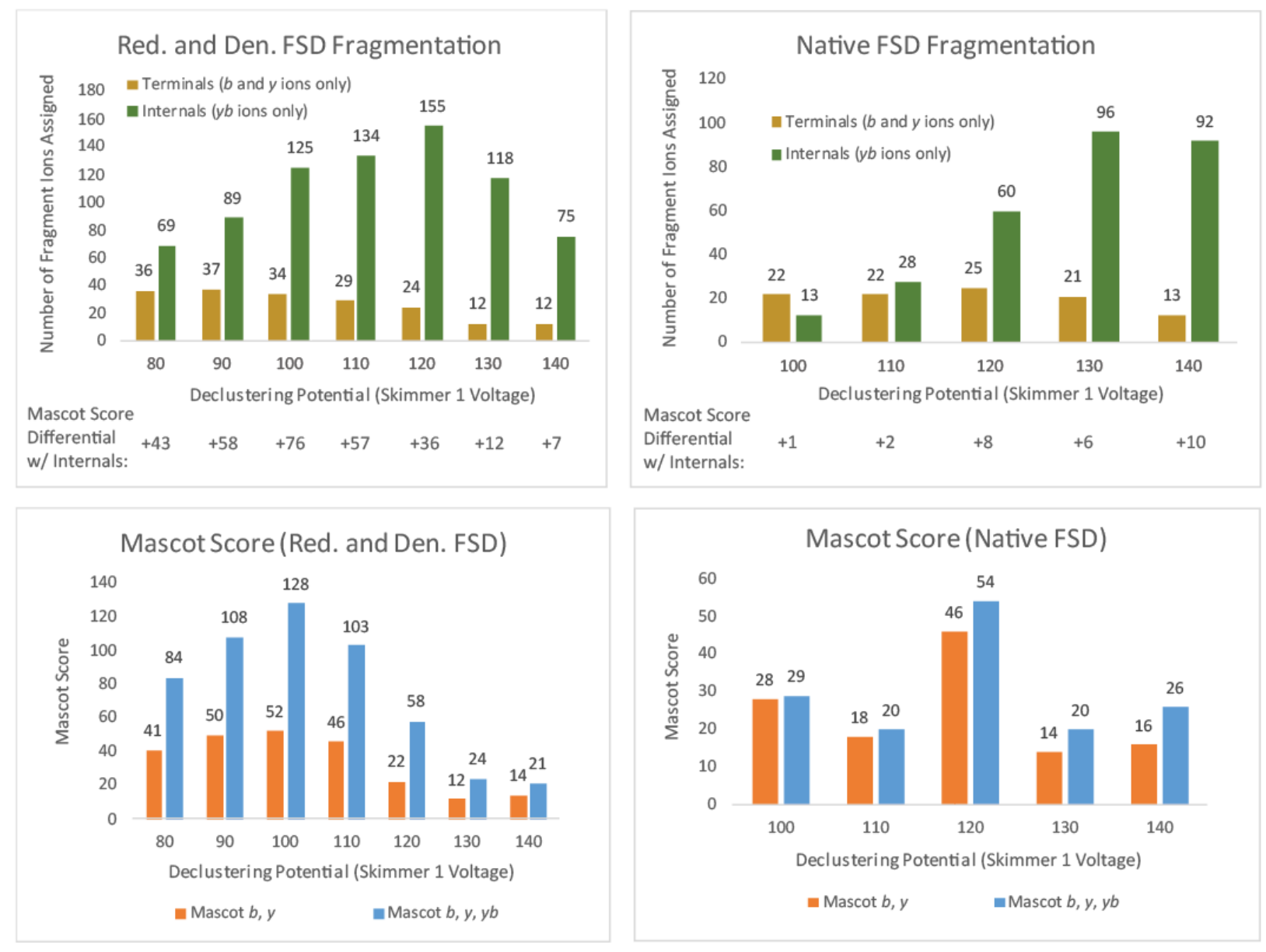

Figure S3. Top: FSD fragmentation of reduced and denatured SOD1G93A at various declustering potentials show that as fragmentation voltage increases, production of terminal fragment ions (gold) decreases slightly, and production of internal fragment ions (green) increases significantly, before beginning to decrease at very high potentials. This phenomenon was also observed for native SOD1 ${ }^{\mathrm{G} 93 \mathrm{~A}}$. Differentials of mascot scores including or ignoring internal $y b$ fragments are shown below each declustering potential value. Bottom: Mascot scores of the corresponding graphs seen above, plotted both with only $b$ and $y$ terminal fragments (orange), and $b$ and $y$ terminal fragments with inclusion of $y b$ internal fragments (blue). 


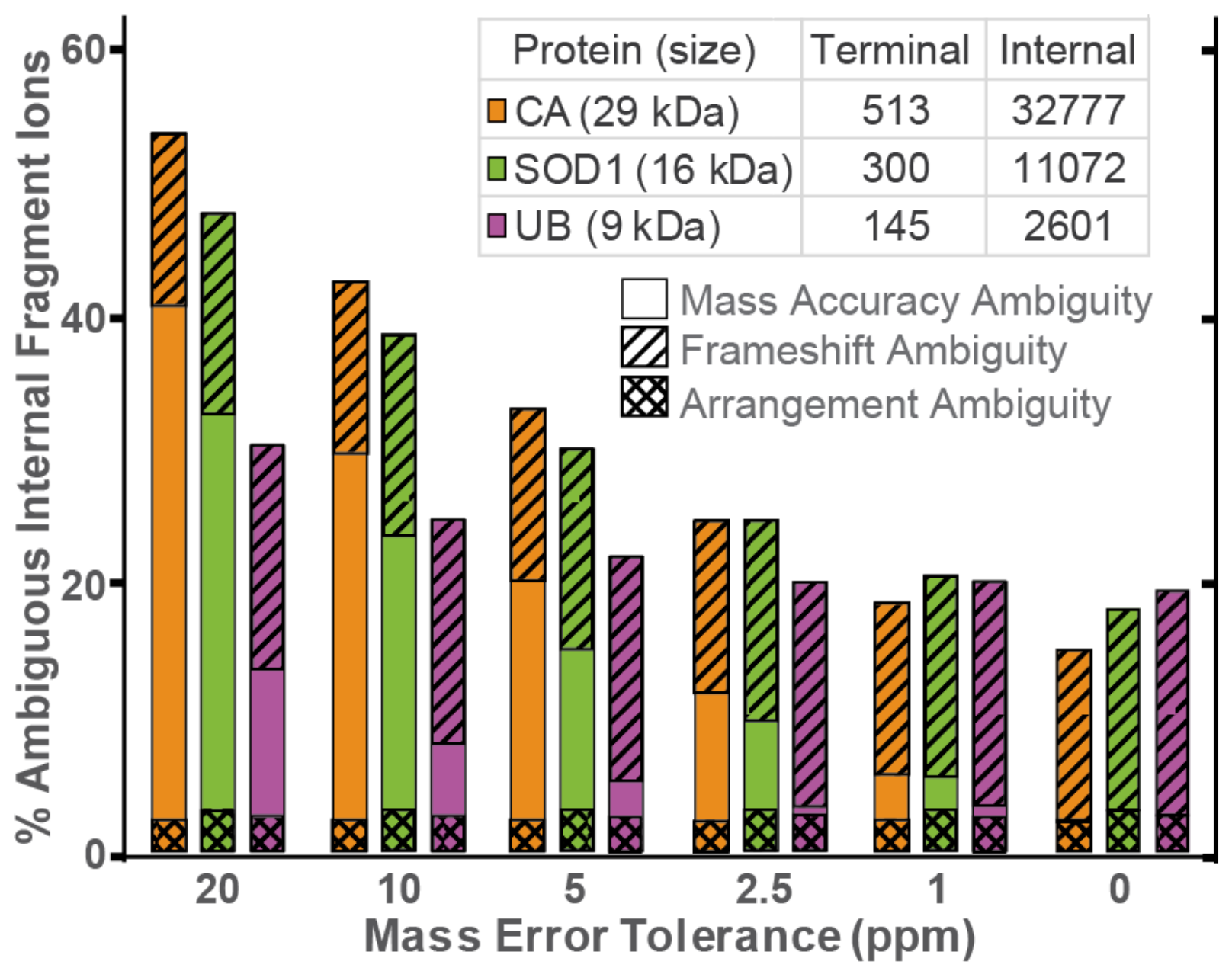

Figure S4. Internal fragment ion ambiguity as a function of protein size, mass accuracy, and ambiguity class. Figure 1 shows this same trend with the frameshift ambiguity removed due to using the frameshift assignment method described. 




B

$$
\mathrm{FA}_{\mathrm{x}}=\mathrm{c}^{2}-\mathrm{c}-2 \mathrm{p}
$$

Frameshift Ambiguity ${ }_{\mathrm{x}}=$ Count $^{2}-$ Count $-(2 \times$ Proximals $)$

$$
\mathrm{FA}_{\text {Total }}=\Sigma \text { All FA }_{\mathrm{x}}
$$

\begin{tabular}{|c|c|c|c|}
\hline & \multicolumn{3}{|c|}{ Ubiquitin (9 kDa) } \\
\hline Residue & Count & Proximals & Frameshift Ambiguity \\
\hline A & 2 & 0 & 2 \\
\hline$R$ & 4 & 1 & 10 \\
\hline $\mathrm{N}$ & 2 & 0 & 2 \\
\hline D & 5 & 0 & 20 \\
\hline C & 0 & 0 & 0 \\
\hline $\mathrm{Q}$ & 6 & 1 & 28 \\
\hline E & 6 & 1 & 28 \\
\hline G & 6 & 1 & 28 \\
\hline $\mathrm{H}$ & 1 & 0 & 0 \\
\hline$I / L$ & 16 & 4 & 232 \\
\hline $\mathrm{K}$ & 7 & 1 & 40 \\
\hline M & 1 & 0 & 0 \\
\hline $\mathrm{F}$ & 2 & 0 & 2 \\
\hline $\mathbf{P}$ & 3 & 1 & 4 \\
\hline s & 3 & 0 & 6 \\
\hline T & 7 & 2 & 38 \\
\hline w & 0 & 0 & 0 \\
\hline $\mathrm{Y}$ & 1 & 0 & 0 \\
\hline $\mathrm{v}$ & 4 & 0 & 12 \\
\hline Total & 76 & 12 & 452 \\
\hline
\end{tabular}

\begin{tabular}{|c|c|c|}
\hline \multicolumn{3}{|c|}{ SOD1-G93A (16 kDa) } \\
\hline Count & Proximals & Frameshift Ambiguity \\
\hline 11 & 1 & 108 \\
\hline 4 & 0 & 12 \\
\hline 7 & 0 & 42 \\
\hline 11 & 2 & 106 \\
\hline 4 & 0 & 12 \\
\hline 3 & 0 & 6 \\
\hline 10 & 2 & 86 \\
\hline 24 & 7 & 538 \\
\hline 8 & 1 & 54 \\
\hline 18 & 4 & 298 \\
\hline 11 & 0 & 110 \\
\hline 0 & 0 & 0 \\
\hline 4 & 0 & 12 \\
\hline 5 & 0 & 20 \\
\hline 10 & 1 & 88 \\
\hline 8 & 1 & 54 \\
\hline 1 & 0 & 0 \\
\hline 0 & 0 & 0 \\
\hline 14 & 3 & 176 \\
\hline & & \\
\hline 153 & 22 & 1722 \\
\hline
\end{tabular}

\begin{tabular}{|c|c|c|}
\hline \multicolumn{3}{|c|}{ Carb. Anhydrase (29 kDa) } \\
\hline Count & Proximals & Frameshift Ambiguity \\
\hline 19 & 4 & 334 \\
\hline 7 & 0 & 42 \\
\hline 17 & 5 & 262 \\
\hline 14 & 2 & 178 \\
\hline 1 & 0 & 0 \\
\hline 9 & 0 & 72 \\
\hline 13 & 0 & 156 \\
\hline 16 & 1 & 238 \\
\hline 11 & 1 & 108 \\
\hline 30 & 4 & 862 \\
\hline 18 & 2 & 302 \\
\hline 2 & 0 & 2 \\
\hline 11 & 2 & 106 \\
\hline 17 & 1 & 270 \\
\hline 30 & 8 & 854 \\
\hline 14 & 0 & 182 \\
\hline 6 & 0 & 30 \\
\hline 8 & 0 & 56 \\
\hline 17 & 2 & 268 \\
\hline & \multicolumn{2}{|c|}{} \\
\hline 260 & 32 & 4322 \\
\hline
\end{tabular}

$\mathrm{D}$ Protein:

\begin{tabular}{|c|c|c|c|}
\hline Protein: & Ubiquitin & SOD1-G93A & Carbonic Anhydrase \\
\hline "0ppm" Ambiguous Internal Fragments: & 509 & 2032 & 4966 \\
\hline Frameshift Ambiguous Fragments: & 452 & 1722 & 4322 \\
\hline Frameshift Ambiguity: & $89 \%$ & $85 \%$ & $87 \%$ \\
\hline Arrangement Ambiguous Fragments: & 57 & 310 & 644 \\
\hline Arrangement Ambiguity: & $11 \%$ & $15 \%$ & $13 \%$ \\
\hline
\end{tabular}

Figure S5. “0 ppm” Ambiguity (frameshift and arrangement, combined) of three model proteins was compared, accounting for how much stems from frameshift ambiguity, due to composition repeats and other events (see note below), and arrangement ambiguity, which collectively includes all other instances of non-mass accuracy ambiguity. A. Depiction of what frameshift ambiguity is and when it applies and does not apply. Fragments whose colors match are have frameshift ambiguity, where whether the fragment is the first or second in the set won't affect which region of the protein is being evaluated. The two R's at the end of the sequence shown are too proximal to generate any frameshift ambiguity if using a low-mass cutoff of $300 \mathrm{Da}$ as we did, or if only considering internal fragments of length 3 or greater. B. Formula for approximating frameshift ambiguity for each residue in a protein. Exact values depend on specific protein sequence. This 
formula uses the two left-most columns of each set in Table $\mathrm{C}$ to calculate the Frameshift ambiguity for each residue. These are then summed to calculate the total frameshift ambiguity for the protein. C. Table for calculating single-residue frameshift ambiguity for each residue set of each protein. This count is unique for all proteins, depends upon the arrangement of amino acids, and can be calculated directly with this method or approximated from only the count of each amino acid without considering the final term in the equation for larger proteins. Notice that leucine and isoleucine are combined here because they are isomers. D. Summary table showing in both total count and percentage how much ambiguity results from frameshift and arrangement ambiguity for these three model proteins. Frameshift ambiguity as a percentage of total ambiguity increases with protein size as a general trend but will always be specific to sequence and degeneracy.

(Note: Repeats of the terminal amino acid result in terminal:internal frameshift ambiguity, which could also be considered if fragmentation energy is not being considered to assign a fragment as a terminal or internal.) 


\begin{tabular}{|c|c|c|c|c|}
\hline & Ubiquitin & SOD1 ${ }^{\mathrm{G93A}}$ & Carbonic Anhydrase & BSA \\
\hline Approximate Size (kDa) & 8.6 & 15.9 & 29.2 & 66.4 \\
\hline Residues & 76 & 153 & 263 & 583 \\
\hline Terminal Fragments & 145 & 300 & 513 & 1159 \\
\hline Internal Fragments & 2601 & 11072 & 32777 & 167841 \\
\hline FS + Arr. Amb. Frags. & 509 & 2032 & 4966 & 27312 \\
\hline FS + Arr. Amb. $\%$ of All lons & $18.5 \%$ & $17.9 \%$ & $14.9 \%$ & $16.2 \%$ \\
\hline Arr. Amb. Frags. & 57 & 310 & 644 & 3502 \\
\hline Arr. Amb. $\%$ of Amb. & $11 \%$ & $15 \%$ & $13 \%$ & $13 \%$ \\
\hline Arr. Amb \% of All lons & $2.1 \%$ & $2.7 \%$ & $1.9 \%$ & $2.1 \%$ \\
\hline FS Amb. Frags. & 452 & 1722 & 4322 & 23810 \\
\hline FS Amb. $\%$ of Amb. & $89 \%$ & $85 \%$ & $87 \%$ & $87 \%$ \\
\hline FS Amb. \% of All lons & $16.5 \%$ & $15.1 \%$ & $13.0 \%$ & $14.1 \%$ \\
\hline 1 ppm Amb. & 528 & 2312 & 6151 & 49847 \\
\hline 1 ppm Amb. \% of All lons & $19.2 \%$ & $20.3 \%$ & $18.5 \%$ & $29.5 \%$ \\
\hline $2.5 \mathrm{ppm}$ Amb. & 532 & 2777 & 8194 & \\
\hline $2.5 \mathrm{ppm}$ Amb. \% of All lons & $19.4 \%$ & $24.4 \%$ & $24.6 \%$ & \\
\hline 5 ppm Amb. & 580 & 3364 & 10975 & \\
\hline 5 ppm Amb. \% of All lons & $21.1 \%$ & $29.6 \%$ & $33.0 \%$ & \\
\hline 10 ppm Amb. & 650 & 4311 & 14052 & \\
\hline 10 ppm Amb. \% of All lons & $23.7 \%$ & $37.9 \%$ & $42.2 \%$ & \\
\hline 20 ppm Amb. & 795 & 5310 & 17750 & \\
\hline \multirow[t]{3}{*}{20 ppm Amb. \% of All lons } & $29.0 \%$ & $46.7 \%$ & $53.3 \%$ & \\
\hline & \multicolumn{2}{|c|}{ After Assigning Frameshift Ambiguit } & & \\
\hline & Ubiquitin & SOD1-G93A & Carbonic Anhydrase & BSA \\
\hline 20 ppm Amb. \% of All lons & $12.5 \%$ & $31.6 \%$ & $40.3 \%$ & \\
\hline 10 ppm Amb. \% of All lons & $7.2 \%$ & $22.8 \%$ & $29.2 \%$ & \\
\hline $5 \mathrm{ppm}$ Amb. \% of All lons & $4.7 \%$ & $14.4 \%$ & $20.0 \%$ & \\
\hline 2.5 ppm Amb. \% of All lons & $2.9 \%$ & $9.3 \%$ & $11.6 \%$ & \\
\hline 1 ppm Amb. \% of All lons & $2.8 \%$ & $5.2 \%$ & $5.5 \%$ & $15.4 \%$ \\
\hline Arr. Amb \% of All lons & $2.1 \%$ & $2.7 \%$ & $1.9 \%$ & $2.1 \%$ \\
\hline
\end{tabular}

Table S1. Calculated ambiguity data on the three model proteins used in this study, with additional statistics on BSA as an exemplar protein approximately twice the size of carbonic anhydrase for scale, calculated up to $1 \mathrm{ppm}$ mass error tolerance. All ambiguities calculated using $300 \mathrm{Da}$ as minimum fragment length. The percentages in the bottom section of the table are plotted in Figure 1, after assignment of frameshift fragments. Abbreviations used: Frags. - Fragments, Amb. Ambiguity, FS - Frameshift, Arr.- Arrangement. 


\begin{tabular}{|c|c|c|c|c|}
\cline { 2 - 5 } \multicolumn{1}{c|}{} & \multicolumn{2}{c|}{ Terminal Fragments Only } & \multicolumn{2}{c|}{ Terminals and Internals } \\
\hline $\begin{array}{c}\text { Before Preprocessing Optimization } \\
\text { Before Frameshift Assignment }\end{array}$ & Native & Reduced & Native & Reduced \\
\hline Residues Observed in Fragments & $22.8 \%$ & $10.5 \%$ & $70.0 \%$ & $88.2 \%$ \\
\hline Sequence Coverage (Cleavage Sites) & $9.9 \%$ & $5.2 \%$ & $48.7 \%$ & $58.0 \%$ \\
\hline Product lons Assigned & 15 & 8 & 92 & 119 \\
\hline $\begin{array}{c}\text { After Preprocessing Optimization } \\
\text { Before Frameshift Assignment }\end{array}$ & Native & Reduced & Native & Reduced \\
\hline Residues Observed in Fragments & $100.0 \%$ & $100.0 \%$ & $100.0 \%$ & $100.0 \%$ \\
\hline Sequence Coverage (Cleavage Events) & $15.1 \%$ & $29.6 \%$ & $59.2 \%$ & $75.0 \%$ \\
\hline Product lons Assigned & 32 & 54 & 97 & 234 \\
\hline $\begin{array}{c}\text { After Preprocessing Optimization } \\
\text { After Frameshift Assignment }\end{array}$ & Native & Reduced & Native & Reduced \\
\hline Residues Observed in Fragments & $100.0 \%$ & $100.0 \%$ & $100.0 \%$ & $100.0 \%$ \\
\hline Sequence Coverage (Cleavage Sites) & $15.1 \%$ & $29.6 \%$ & $64.5 \%$ & $80.9 \%$ \\
\hline Product lons Assigned & 32 & 54 & 157 & 344 \\
\hline
\end{tabular}

Table S2. Fragmentation coverage and ions generated when using terminal fragments alone vs. using terminals and internals. Results shown at three indicated steps in acquisition and assignment process. Key improvements of preprocessing include correcting repetitive building block from Averagine to exact protein composition, tuning of quality factor threshold to achieve a lower-than $1 \%$ false positive rate, and applying an internal calibration to commonly-observed $b$ ions. Assigning previously ambiguous frameshift assignments accounted for a 1.62- and 1.47-fold increase in product ions assigned for native and reduced, respectively. 